\title{
$H(z)$ diagnostics on the nature of dark energy
}

\author{
Sergio del Campo* and Ramón Herrera ${ }^{\dagger}$ \\ Instituto de Física, Pontificia Universidad Católica de Valparaíso, \\ Avenida Brasil 2950, Casilla 4059, Valparaíso, Chile \\ Diego Pavón $\ddagger$ \\ Departamento de Física, Facultad de Ciencias, \\ Universidad Autónoma de Barcelona, \\ 08193 Bellaterra (Barcelona), Spain
}

\begin{abstract}
The two dominant components of the cosmic budget today, pressureles matter and dark energy, may or may not be interacting with each other. Currently, both possibilities appear compatible with observational data. We propose several criteria based on the history of the Hubble factor that can help discern whether they are interacting and whether dark energy is phantom or quintessence in nature.
\end{abstract}

PACS numbers:

\footnotetext{
* E-mail address: sdelcamp@ucv.cl

$\dagger$ E-mail address: ramon.herrera@ucv.cl

¥ E-mail address: diego.pavon@uab.es
} 


\section{INTRODUCTION}

Recently, Sahni et al. used the luminosity distance $d_{L}=c(1+z) \int_{0}^{z} d z^{\prime} / H\left(z^{\prime}\right)$, valid in spatially flat Friedmann-Robertson-Walker (FRW) universes, to propose a diagnostic to tell the cosmological constant from evolving dark energy fields [1]. For universes dominated by cold matter (CM), subscript $m$, and dark energy (DE), subscript $d$, with constant equation of state, $w=p_{d} / \rho_{d}<-1 / 3$, Friedmann's equation takes the form

$$
E^{2}(x)=\Omega_{m 0} x^{3}+\Omega_{d 0} x^{3(1+w)},
$$

where $x:=1+z=a_{0} / a$, and $E(x):=H(x) / H_{0}$ is the normalized Hubble function. In writing this expression it was implicitly assumed that cold dark matter (CDM) and DE evolved separately. As usual, $\Omega_{i 0}:=\kappa^{2} \rho_{i 0} /\left(3 H_{0}^{2}\right)$ with $i=m, d$ stand for the energy densities of the components in units of the critical density, $\kappa^{2}:=8 \pi G$, and a zero subscript denotes the present-day value of the corresponding quantity.

Sahni et al. [1] introduced the redshift dependent function

$$
O m(x):=\frac{E^{2}(x)-1}{x^{3}-1},
$$

which for spatially flat FRW cosmologies $\left(\Omega_{m 0}+\Omega_{d 0}=1\right)$ reduces to $\operatorname{Om}(x)=\Omega_{m 0}+$ $\left(1-\Omega_{m 0}\right)\left(x^{3(1+w)}-1\right)\left(x^{3}-1\right)^{-1}$. It has the interesting feature that for the $\Lambda$ CDM model $\left(\rho_{d}=\right.$ constant, $\left.w=-1\right)$, it yields $O m(x)=\Omega_{m 0}$, whereas for quintessence fields $(-1<w<$ $-1 / 3)$ and phantom fields $(w<-1)$ it gives $O m(x)>\Omega_{m 0}$ and $O m(x)<\Omega_{m 0}$, respectively.

Since this diagnostic does not depend on the present values of the density parameters, $\Omega_{m 0}$ and $\Omega_{d 0}$, it circumvents the drawbacks brought about by our comparatively poor knowledge of them. In fact, it is expected to be useful when accurate data of $H$ at different redshifts become available. This seems more feasible than to substantially upgrade our knowledge of $\Omega_{m 0}$ and $\Omega_{d 0}$ in the near future.

At present, the $\Lambda \mathrm{CDM}$ model fits the observational data, within statistical errors, rather well -see, e.g. [2]. Nonetheless if future data come to reveal that $\operatorname{Om}(x) \neq \Omega_{m 0}$, a number of DE alternatives should be considered including scenarios in which the DE interacts nongravitationally with other components.

Here, we assume that DE and CDM, subscript $c$, interact with each other (whence they do not evolve separately) but not with baryonic matter, subscript $b$, since interactions with 
baryons are strongly constrained by experiments [3]. This possibility is being actively considered in the literature -see [4] and references therein- since it is rather natural [5, 6] and more general than otherwise, it substantially alleviates the coincidence problem (i.e., "why are the densities of matter and dark energy of the same order precisely today"), and agrees with observation.

In this paper we propose several criteria, based on the evolution of the Hubble factor, to help discriminate models in which DE and CDM interact with each other non-gravitationally from models in which they do not, and to tell quintessence DE energy models from phantom models. Clearly, for these criteria to be useful accurate data of $H(z)$ are needed. While the present data exhibit large error bars [7-9] the situation may well improve comparatively soon thanks to upcoming and planned experiments. The Baryon Oscillation Spectroscopy Survey (BOSS) 5-years project [10] aims at measuring the absolute cosmic distance scale and expansion rate with percent-level precision at redshifts $z<0.7$ and $z \simeq 2.5$ using the standard rule furnished by the baryon acoustic oscillations. The forecast precision for $H(z)$ at $z=0.35,0.6$ and 2.5 is $1.8 \%, 1.7 \%$ and $1.2 \%$, respectively. Data at intermediate and larger redshifts will partly come by adapting to $z>0$ the method proposed by Hogan [11] which makes use of the gravitational radiation emitted by black hole binary inspiral events to determine $H_{0}$ with a precision better than $1 \%$. The method may be extended up to $z \sim 1$ redshifts, and even to $z \simeq 10$ by observing massive black hole binaries since there will likely be a direct electromagnetic counterpart of the merger [12]. An even more promising method, proposed by Corasaniti et al. [13] to constrain cosmological parameters, exploits the Sandage-Loeb test [14]. It consists in measuring the redshift of distant objects at two separate times, say $t_{0}$ and $t_{0}+\Delta t_{0}$, at the present epoch. The redshift variation of the source (subscript $s$ ) between these two instants is, at a very good precision, $\Delta z_{s} \simeq$ $\left(\dot{a}\left(t_{0}\right)-\dot{a}\left(t_{s}\right)\right) / a\left(t_{s}\right)$-see Eq. (5) in [13]. This expression, re-written as $H\left(z_{s}\right) \simeq\left(\Delta z_{s} / \Delta t_{0}\right)-$ $H_{0}\left(1+z_{s}\right)$, provides us with the value of the Hubble factor at the time the source emitted the electromagnetic signal that is currently arriving at our detectors (either telescopes or radio-telescopes), modulo we know $H_{0}$ accurately enough. The authors of [13] suggested using the absorption lines of the Lyman- $\alpha$ forest in the redshift interval $2 \leq z \leq 5$ and $\Delta t_{0}=10$ years.

Altogether, encouraged by the prospects of a greatly improved knowledge of the history of $H(z)$ we present several criteria that may help telling apart interacting from noninteracting 
dark energy models.

Section II presents on phenomenological basis some possible expressions of the interaction term. Sections III and IV introduce various criteria to distinguish interacting from noninteracting DE models. Section $\mathrm{V}$ focus on the statefinder parameters and compares their usefulness with the criteria based on the Hubble history. Finally, section VI summarizes our findings.

\section{EXPRESSIONS FOR THE INTERACTION BETWEEN DARK ENERGY AND DARK MATTER}

Since the dark components are assumed to interact with one another also nongravitationally but not with baryons the conservation equations take the form

$$
\begin{aligned}
& \dot{\rho}_{b}+3 H \rho_{b}=0, \\
& \dot{\rho}_{c}+3 H \rho_{c}=Q, \\
& \dot{\rho}_{d}+3 H(1+w) \rho_{d}=-Q,
\end{aligned}
$$

where $Q$ denotes the interaction term and the relationship $\rho_{b}+\rho_{c}=\rho_{m}$ is understood. It is worth noticing that when $Q>0$-as we shall consider throughout- the coincidence problem is alleviated since $\rho_{c}$ decreases more slowly with expansion and $\rho_{d}$ more quickly, and the problem may even be solved in full [15]. On the other hand, if $Q$ were negative, the second law of thermodynamics could be violated [16], and the energy densities of CDM or DE could become negative at high or low redshifts.

In the absence of a fundamental theory for dark energy the quantity $Q$ cannot be derived from first principles. However, we may guess likely expressions for it by noting that $Q$ must

be small [17] (at least lower than $3 H \rho_{m}$ ) and depend on the energy densities multiplied by a quantity with units of inverse of time, a rate. We shall deal with two different possibilities considered in the literature, namely, $(i)$ that the said rate is proportional to the Hubble factor, and (ii) that it is just a constant [18].

In case $(i)$, we have that $Q=Q\left(H \rho_{c}, H \rho_{d}\right)$. By power law expanding this function and retaining just the first term one follows $Q \simeq \epsilon_{c} H \rho_{c}+\epsilon_{d} H \rho_{d}$. Given the lack of information about the coupling, it appears advisable to work with just one parameter rather than two. Thus, three different choices arise, namely, $\epsilon_{c}=0, \epsilon_{d}=0$, and $\epsilon_{c}=\epsilon_{d}$. It is worth 
mentioning that expressions of this type can be obtained from the scalar-tensor theory of gravity developed by Kaloper et al. [19] -see e.g. [20, 21]. In the next section we shall consider successively three expressions for $Q$ with $0<\epsilon<<1$, while keeping $w=$ constant throughout, and look for diagnostics -based in the history of $H(z)$ that may tell interacting from noninteracting models.

In case $(i i)$ we have $Q=Q\left(\rho_{c}, \rho_{d}\right)$ and proceeding as before we write

$$
Q=3\left(\Gamma_{c} \rho_{c}+\Gamma_{d} \rho_{d}\right)
$$

where the $\Gamma_{i}(i=c, d)$ quantities denote constant rates. This expression was inspired in reheating models [22], curvaton decay [23], and decay of DM into radiation [24]. Regrettably, one of the energy densities $\left(\rho_{m}\right.$ or $\left.\rho_{d}\right)$ becomes negative either at early or late times [18] and, generally, it does not solve the coincidence problem. Nevertheless, we will briefly consider it in the section after next.

\section{INTERACTION TERM $Q=Q\left(H \rho_{c}, H \rho_{d}\right)$}

We begin by assuming that the interaction term takes the form

$$
Q=3 \epsilon H \rho_{c},
$$

where the factor 3 was introduced for mathematical convenience.

\section{A. Decaying cosmological constant}

One way to explain the present small value of the cosmological constant is to assume a transfer of energy from the quantum vacuum to particles and/or radiation -i.e., a decaying cosmological constant (DCC). Along the years a diversity of phenomenological models have been proposed, many of which are summarized in Ref. [25]. If the quantum vacuum $(w=$ -1) slowly decays into non-baryonic CDM particles, integration of Eqs. (3)-(5) yields

$$
\begin{aligned}
\rho_{b} & =\rho_{b 0} x^{3} \\
\rho_{c} & =\rho_{c 0} x^{3(1-\epsilon)} \\
\rho_{\Lambda} & =\rho_{\Lambda 0}+\left(\frac{\epsilon}{1-\epsilon}\right) \rho_{c 0}\left(x^{3(1-\epsilon)}-1\right) .
\end{aligned}
$$


This kind of models are not purely phenomenological any more for they have received some persuasive backings from the theoretical side [26-30], which makes their consideration all the more interesting. In particular, by means of the renormalization group theory the authors of [27] arrive to a simple expression for $\epsilon$ ( $\nu$ in their notation) in terms of the masses of the super-heavy sub-planckian particles -see their Eq. (4.10). A rather similar expression was found by removing the flat space-time contribution to the vacuum energy of quantum fields in homogeneous and isotropic universes by employing a technique similar to that used in the computation of the Arnowitt-Deser-Misner mass -see Eq. (39) in Ref. [29]. Moreover, consistency both with primordial nucleosynthesis and large scale structure formation leads to the constraint $|\epsilon| \ll 1$ [27], [31]. Recently, DCC models were revisited by Basilakos et al. [32] and, for some specific choices of $\Lambda(t)$, confronted with observational data from supernovae type Ia, baryon acoustic oscillations, the cosmic microwave background shift parameter, and the growth rate of galaxy clustering.

As said above, the coincidence problem gets alleviated since

$$
\left(\frac{|\dot{r} / r|_{D C C}}{|\dot{r} / r|_{\Lambda C D M}}\right)_{0}=1-\epsilon\left[\left(\Omega_{c} / \Omega_{\Lambda}\right)_{0}+r_{0}\right]<1,
$$

i.e., currently the ratio $r \equiv\left(\rho_{b}+\rho_{c}\right) / \rho_{\Lambda}$ varies more slowly than in the $\Lambda$ CDM model. This also holds true for the other interaction terms considered below.

Using Friedmann's equation, $3 H^{2}=\kappa^{2}\left[\rho_{b}+\rho_{c}+\rho_{\Lambda}\right]$, we can write

$$
E^{2}(x)=\Omega_{b 0} x^{3}+\Omega_{c 0} x^{3(1-\epsilon)}+\Omega_{\Lambda 0}+\left(\frac{\epsilon}{1-\epsilon}\right) \Omega_{c 0}\left[x^{3(1-\epsilon)}-1\right],
$$

where $\Omega_{\Lambda 0}=1-\Omega_{b 0}-\Omega_{c 0}$. Obviously, in this case $O m(x) \neq \Omega_{m 0}$ with $\Omega_{m 0}=\Omega_{b 0}+\Omega_{c 0}$, but rather

$$
O m(x)=\Omega_{b 0}+\frac{\Omega_{c 0}}{1-\epsilon} \frac{x^{3(1-\epsilon)}-1}{x^{3}-1} .
$$

Thus, the $O m(x)$ criterion would mistake a DCC model by a quintessence field (phantom field) if applied at low redshifts (high redshifts). Similar situations occur if the said criterion is used on models considered below in this paper.

The first and second derivatives of $E^{2}(x)$ with respect to $x^{3}$ are $d E^{2}(x) / d x^{3}=\Omega_{b 0}+\Omega_{c 0} x^{-3 \epsilon}$, and $d^{2} E^{2}(x) / d\left(x^{3}\right)^{2}=-\epsilon \Omega_{c 0} x^{-3(1+\epsilon)}$, respectively. Therefore, for large redshifts the first derivative tends to $\Omega_{b 0}$ from above. Likewise, $E^{2}(x)$ is concave (i.e., $\left.d^{2} E^{2}(x) / d\left(x^{3}\right)^{2}<0\right)$. By contrast, for noninteracting dark energy fields with $w=$ constant -see Eq. (11)- one has, $d E^{2}(x) / d x^{3}=\Omega_{m 0}+\left(1-\Omega_{m 0}\right)(1+w) x^{3 w}$. For large 
redshifts this expression tends to $\Omega_{m 0}$ from above if $\mathrm{DE}$ is of quintessence type and from below if it is phantom. In view that, observationally, $\Omega_{b 0}$ and $\Omega_{m 0}$ are separated by a non small gap (the latter might be about six or seven times larger than the former) this may serve discriminate DCC models from noninteracting quintessence and phantom models. One may think that we would have arrived to the same result just just by dividing $E^{2}(x)$ by $x^{3}$; i.e., $E^{2}(x) / x^{3}=\Omega_{m 0}+\left(1-\Omega_{m 0}\right) x^{3 w}$. However, this is not true because of the factor $(1+w)$ in the second term of the derivative which makes the latter tend to $\Omega_{m 0}$ faster, and it is key to tell whether the DE is quintessence's or phantom's type.

As a corollary, we may say that the sign of the second derivative, may discriminate DCC from noninteracting phantom models, the former being negative and the latter positive, $d^{2} E^{2}(x) / d\left(x^{3}\right)^{2}=\left(1-\Omega_{m 0}\right) w(1+w) x^{3(w-1)}$; only that this criterion can be used also at smaller redshift than the preceding one.

Likewise, by measuring $H(z)$ at two separated redshifts, $z_{1}$ and $z_{2}$ with $z_{1}<z_{2}$, they may be discriminated. Indeed, for noninteracting phantom models the quantity $\Delta\left(x_{1}, x_{2}\right):=$ $E^{2}\left(x_{2}\right)-E^{2}\left(x_{1}\right)$ is

$$
\Delta\left(x_{1}, x_{2}\right)=\Omega_{m 0}\left[x_{2}^{3}\left(1-x_{2}^{3 w}\right)-x_{1}^{3}\left(1-x_{1}^{3 w}\right)\right]+x_{2}^{3(1+w)}-x_{1}^{3(1+w)},
$$

and it can be negative if the redshifts are sufficiently apart. By contrast, the corresponding quantity for DCC models,

$$
\Delta\left(x_{1}, x_{2}\right)=\Omega_{b 0}\left(x_{2}^{3}-x_{1}^{3}\right)+\Omega_{c 0}\left(\frac{1}{1-\epsilon}\right)\left[x_{2}^{3(1-\epsilon)}-x_{1}^{3(1-\epsilon)}\right],
$$

will always be positive-definite.

Let us assume that, thanks to extra information, we come to know that the right model must be a DCC one. Note that to discriminate which one (i.e., its $\epsilon$ value) neither direct use of last expression nor of Eq. (9) will prove very useful, given the comparatively wide uncertainty in the value of $\Omega_{c 0}$. However, the following strategy may work. Consider two competing DCC models (say A and B) and recall that $\Omega_{b 0}$ is reasonably well determined from primordial nucleosynthesis $\left(0.036 \leq \Omega_{b 0} \leq 0.047\right.$, see, e.g. Ref. [33]) together with the present value of the Hubble factor. The ratio

$$
R_{\Delta}:=\frac{\left[\Delta\left(x_{1}, x_{2}\right)-\Omega_{b 0}\left(x_{2}^{3}-x_{1}^{3}\right)\right]_{A}}{\left[\Delta\left(x_{1}, x_{2}\right)-\Omega_{b 0}\left(x_{2}^{3}-x_{1}^{3}\right)\right]_{B}}=\left(\frac{1-\epsilon_{B}}{1-\epsilon_{A}}\right) \frac{x_{2}^{3\left(1-\epsilon_{A}\right)}-x_{1}^{3\left(1-\epsilon_{A}\right)}}{x_{2}^{3\left(1-\epsilon_{B}\right)}-x_{1}^{3\left(1-\epsilon_{B}\right)}}
$$

depends on $\epsilon_{A}$ and $\epsilon_{B}$ only. This will enable us to determine which DCC model, A or B, fits better the $H(z)$ data. 
It should be noted that, in this regard, it looks more advantageous to use this other ratio

$$
R_{O m}:=\frac{O m\left(x_{2}\right)-\Omega_{b 0}}{O m\left(x_{1}\right)-\Omega_{b 0}}=\frac{x_{1}^{3}-1}{x_{2}^{3}-1} \frac{x_{2}^{3(1-\epsilon)}-1}{x_{1}^{3(1-\epsilon)}-1},
$$

since it can constrain $\epsilon$ more easily.

At any rate, aside from this specific instance, the $O m(x)$ function (Eq. (2)) is not much useful when dealing with interacting models since, in these models, CDM and baryonic matter redshift at different rates with expansion whereby the current fractional densities, $\Omega_{b 0}$ and $\Omega_{c 0}$, enter the expression for $E^{2}$ multiplied by different powers of $x$. This explains the restricted application range of the said function and we will not resort to it any more in this paper.

\section{Decaying dark energy field}

For the more general case $w=$ constant $\neq-1$, one has

$$
\begin{aligned}
& \rho_{b}=\rho_{b 0} x^{3} \\
& \rho_{c}=\rho_{c 0} x^{3(1-\epsilon)}, \\
& \rho_{d}=\rho_{d 0} x^{3(1+w)}+\left(\frac{\epsilon}{\epsilon+w}\right) \rho_{c 0}\left[x^{3(1+w)}-x^{3(1-\epsilon)}\right] .
\end{aligned}
$$

Thereby

$$
E^{2}(x)=\Omega_{b 0} x^{3}+\Omega_{c 0} x^{3(1-\epsilon)}+\Omega_{d 0} x^{3(1+w)}+\left(\frac{\epsilon}{\epsilon+w}\right) \Omega_{c 0}\left[x^{3(1+w)}-x^{3(1-\epsilon)}\right],
$$

being

$$
\begin{aligned}
\frac{d E^{2}(x)}{d x^{3}} & =\Omega_{b 0}+\Omega_{c 0}(1-\epsilon) x^{-3 \epsilon}+\Omega_{d 0}(1+w) x^{3 w} \\
& +\left(\frac{\epsilon}{\epsilon+w}\right) \Omega_{c 0}\left[(1+w) x^{3 w}-(1-\epsilon) x^{-3 \epsilon}\right]
\end{aligned}
$$

and

$$
\begin{aligned}
\frac{d^{2} E^{2}(x)}{d\left(x^{3}\right)^{2}} & =-(1-\epsilon) \Omega_{c 0} x^{-3(1+\epsilon)}+w(1+w) \Omega_{d 0} x^{3(w-1)} \\
& +\left(\frac{\epsilon}{\epsilon+w}\right) \Omega_{c 0}\left[w(1+w) x^{3(w-1)}+\epsilon(1-\epsilon) x^{-3(1+\epsilon)}\right]
\end{aligned}
$$

In view of the hypothesis $0<\epsilon<<1$ and the fact that observation reveals that $|1+w|<<$ 1, the right hand side of (17) is dominated by its two first terms. Likewise, the first term 
dominates the right hand side of (18). Therefore, for not large redshifts (i.e., $z<3$ ) the normalized Hubble function, $E^{2}(x)$, is growing and concave. In consequence, its shape will not tell a decaying energy field (with $w \neq-1$ ) from DCC models or from noninteracting quintessence or phantom models with constant equation of state. However, discrimination will likely come at higher redshifts since, as before, the first derivative may tell interacting from noninteracting cosmologies depending on whether $d E^{2}(x) / d x^{3}$ tends to $\Omega_{b 0}$ or $\Omega_{c 0}$ at large redshifts.

Figure 11 shows the evolution of the second derivative of $E^{2}(x)$, as given by Eq. (18), for the best fit values of $w$ and $\epsilon$ of model III of He et al. [34] (dotted line) as constrained by data from supernovae, baryon acoustic oscillations, cosmic microwave background, and the value of the Hubble constant, $H_{0}$. Also shown is the noninteracting case (solid line). The fact that both lines nearly overlap emphasizes the need for accurate data of $H(z)$.

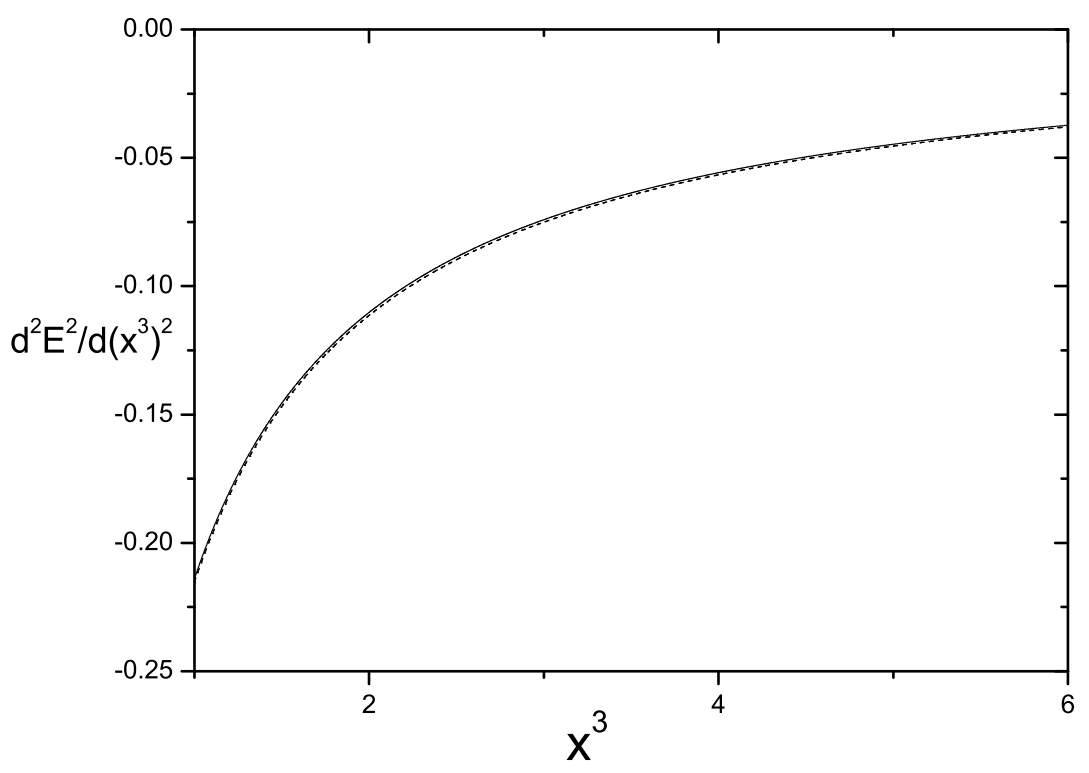

FIG. 1: Dotted line: plot of the second derivative of $E^{2}$ in terms of $x^{3}$ for the best fit values, $w=-1.02, \epsilon=0.0006$, with $\Omega_{c 0}=0.230$, and $\Omega_{d 0}=0.725$ of model III, $Q=3 H \epsilon \rho_{c}$, of Ref. [34]. Solid line: same as before but with $\epsilon=0$ (no interaction). 
In the present case

$$
\begin{aligned}
\Delta\left(x_{1}, x_{2}\right) & =\Omega_{b 0}\left(x_{2}^{3}-x_{1}^{3}\right)+\Omega_{c 0}\left[x_{2}^{3(1-\epsilon)}-x_{1}^{3(1-\epsilon)}\right]+\Omega_{d 0}\left[x_{2}^{3(1+w)}-x_{1}^{3(1+w)}\right] \\
& +\left(\frac{\epsilon}{\epsilon+w}\right) \Omega_{c 0}\left[x_{2}^{3(1+w)}-x_{1}^{3(1+w)}-x_{2}^{3(1-\epsilon)}+x_{1}^{3(1-\epsilon)}\right] .
\end{aligned}
$$

For $0<\epsilon<<1$ and $1+w \simeq 0$ the two last terms are subdominants, and the ratio defined by the first equality in (13) comes to be

$$
R_{\Delta} \simeq \frac{x_{2}^{3\left(1-\epsilon_{A}\right)}-x_{1}^{3\left(1-\epsilon_{A}\right)}}{x_{2}^{3\left(1-\epsilon_{B}\right)}-x_{1}^{3\left(1-\epsilon_{B}\right)}} .
$$

Thus, thanks to the factor $\left(1-\epsilon_{B}\right) /\left(1-\epsilon_{A}\right)$ on the right hand side of Eq. (13) we hope discriminate DCC models from interacting dark energy models with $w$ close to the cosmological constant value, -1 , and also discriminate within models featuring the interaction (7).

\section{B. Interaction term proportional to $H \rho_{d}$}

We now assume that the interaction term takes the form

$$
Q=3 \epsilon H \rho_{d}
$$

which may be key to explain the non-vanishing temperature of sterile neutrinos [35], [36].

Then, the conservation equations (3)-(44) integrate to

$$
\begin{aligned}
& \rho_{b}=\rho_{b 0} x^{3} \\
& \rho_{c}=\rho_{c 0} x^{3}+\left(\frac{\epsilon}{w+\epsilon}\right) \rho_{d 0}\left[1-x^{3(w+\epsilon)}\right] x^{3}, \\
& \rho_{d}=\rho_{d 0} x^{3(1+w+\epsilon)} .
\end{aligned}
$$

Hence,

$$
E^{2}(x)=x^{3}-\Omega_{d 0}\left(\frac{w}{w+\epsilon}\right)\left[x^{3}-x^{3(1+w+\epsilon)}\right] .
$$

For $w=-1$ (i.e., DCC with interaction given by Eq. (21)), it reduces to

$$
E^{2}(x)=x^{3}-\frac{\Omega_{\Lambda 0}}{1-\epsilon}\left[x^{3}-x^{3 \epsilon}\right] \text {, }
$$

which is to be compared with (9). Again, $E^{2}(x)$ is growing and concave whence its graph will tell neither DCC models with interaction term given by (21) from DCC models with 
interaction term given by (7) nor these models from noninteracting quintessence models with $w=$ constant

Expression (24) will help constrain $\epsilon$ without need of knowing $\Omega_{\Lambda 0}$ since the ratio

$$
\frac{\left[E^{2}(x)-x^{3}\right]_{x=x_{1}}}{\left[E^{2}(x)-x^{3}\right]_{x=x_{2}}}=\frac{x_{1}^{3}-x_{1}^{3 \epsilon}}{x_{2}^{3}-x_{2}^{3 \epsilon}}
$$

does not depend on that quantity.

Unlike the two previous cases, as inspection of the right hand side of Eq. (23) shows, the behavior of the derivative $d E^{2}(x) / d x^{3}$ at high redshifts will not discriminate interacting from noninteracting cosmological models. For the discrimination to be possible $\epsilon$ should take unrealistic high values.

By deriving twice (23) we get

$$
\frac{d^{2} E^{2}(x)}{d\left(x^{3}\right)^{2}}=\Omega_{d 0} w(1+w+\epsilon) x^{3(w+\epsilon-1)} .
$$

When this latter quantity gets accurately determined, we will be able to set useful constraints on $w$ and $\epsilon$ since its ratio for two decaying dark energy (DDE) models (say $A$ and $B$ ) does not depend on the $\Omega$ parameters,

$$
\frac{d^{2} E^{2} /\left.d\left(x^{3}\right)^{2}\right|_{A}}{d^{2} E^{2} /\left.d\left(x^{3}\right)^{2}\right|_{B}}=\frac{w_{A}\left(1+w_{A}+\epsilon_{A}\right)}{w_{B}\left(1+w_{B}+\epsilon_{B}\right)} \frac{x^{3\left(w_{A}+\epsilon_{A}\right)}}{x^{3\left(w_{B}+\epsilon_{B}\right)}} .
$$

Once again $E^{2}(x)$, given by (23), is a growing function of redshift, concave for quintessence fields and convex for phantom fields. Accordingly, the shape of $E^{2}(x)$ can tell decaying quintessence fields from decaying phantom fields but not from noninteracting DE fields.

Figure 2 depicts the evolution of the second derivative of $E^{2}(x)$, as given by Eq. (26)), for the best fit values of $w$ and $\epsilon$ of model II of Ref. [34] (dotted line) as constrained by observational data. The solid line corresponds to the noninteracting case (solid line). As it is apparent the lower the redshift, the easier the discrimination.

\section{Interaction term proportional to $H\left(\rho_{c}+\rho_{d}\right)$}

Assuming the interaction term

$$
Q=3 \epsilon H\left(\rho_{c}+\rho_{d}\right)
$$




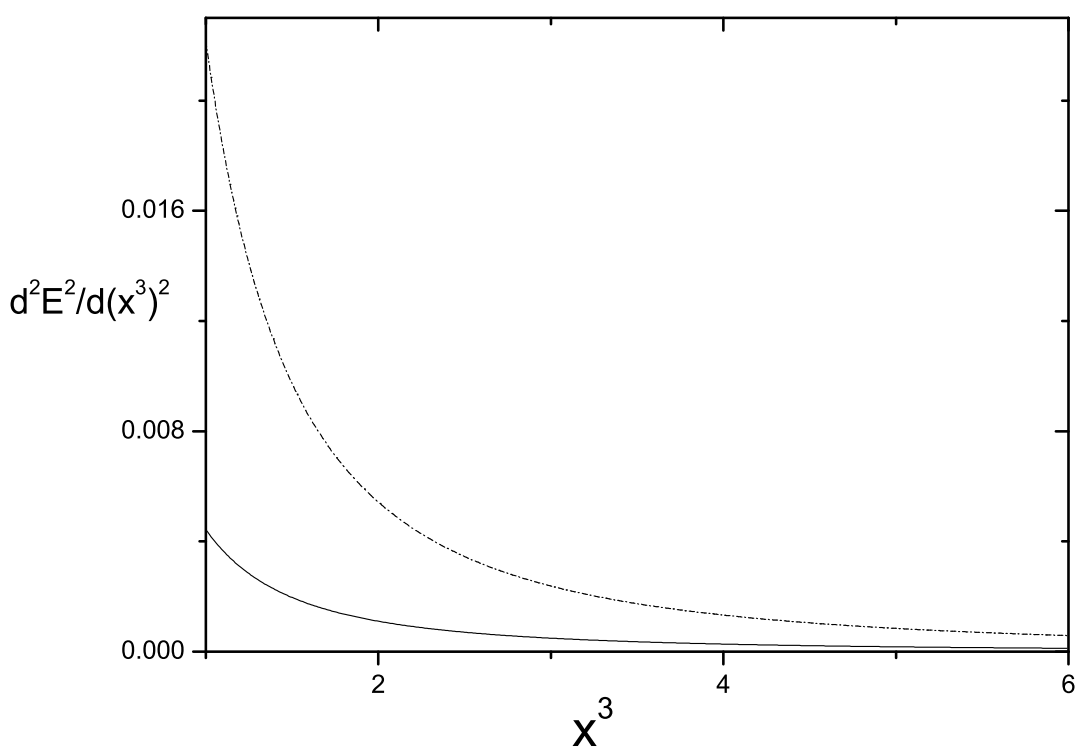

FIG. 2: Dotted line: plot of the second derivative of $E^{2}$ in terms of $x^{3}$ for the best fit values, $w=-1.03, \epsilon=0.024$, with $\Omega_{d 0}=0.717$ of model II, $Q=3 H \epsilon \rho_{d}$, of Ref. [34]. Solid line: same as before but with $\epsilon=0$ (no interaction).

widely considered in the literature -see, e.g. [15, 18, 37]-, equations (3)-(5) integrate to

$$
\begin{aligned}
\rho_{b} & =\rho_{b 0} x^{3} \\
\rho_{c} & =C_{1} x^{\gamma_{1}}+C_{2} x^{\gamma_{2}}, \\
\rho_{d} & =\frac{1}{2 \epsilon}\left[-C_{1}(A+B)+C_{2}(B-A) x^{-3 B}\right] x^{\gamma_{1}},
\end{aligned}
$$

respectively.

Here

$$
\begin{array}{cc}
\gamma_{1}=\frac{3}{2}[2+w+\sqrt{w(w+4 \epsilon)}], & \gamma_{2}=\frac{3}{2}[2+w-\sqrt{w(w+4 \epsilon)}] \\
C_{1}=\frac{1}{2 B}\left[(B-A) \rho_{c 0}-2 \epsilon \rho_{d 0}\right], & C_{2}=\frac{1}{2 B}\left[(A+B) \rho_{c 0}+2 \epsilon \rho_{d 0}\right], \\
A=(w+2 \epsilon), \quad \text { and } & B=\sqrt{w(w+4 \epsilon)} .
\end{array}
$$

As in the two previous cases, the three energy densities remain semi-positive definite for all $x$. Therefore,

$$
E^{2}(x)=\Omega_{b 0} x^{3}+\frac{1}{2 \epsilon}\left\{\Omega_{C_{1}}[2 \epsilon-(A+B)]-\Omega_{C_{2}}(A-B) x^{-3 B}\right\} x^{\gamma_{1}}+\Omega_{C_{2}} x^{\gamma_{2}},
$$


where

$$
\Omega_{C_{1}}=\frac{1}{2 B}\left[\Omega_{c 0}(B-A)-2 \epsilon \Omega_{d 0}\right], \quad \text { and } \quad \Omega_{C_{2}}=\frac{1}{2 B}\left[\Omega_{c 0}(A+B)+2 \epsilon \Omega_{d 0}\right]
$$

also stays non-negative as it should. Further, $E^{2}$ is a growing function of $x^{3}$, concave for quintessence and convex for phantom -see Fig. 3. which will help discriminate phantom from quintessence DE in interacting models with $Q$ given by Eq. (28). However, as is apparent, for $x^{3} \geq 5$ the graphs show a nearly straight line behavior whereby one must focus on redshifts below, say, 1.7 .

Inspection of the right hand side of (30) shows that for interacting models $(\epsilon>0)$ the derivative $d E^{2}(x) / d x^{3}$ tends to $\Omega_{b 0}$ at high redshifts. Accordingly if observation reveals that behavior, it would be suggestive of interaction.
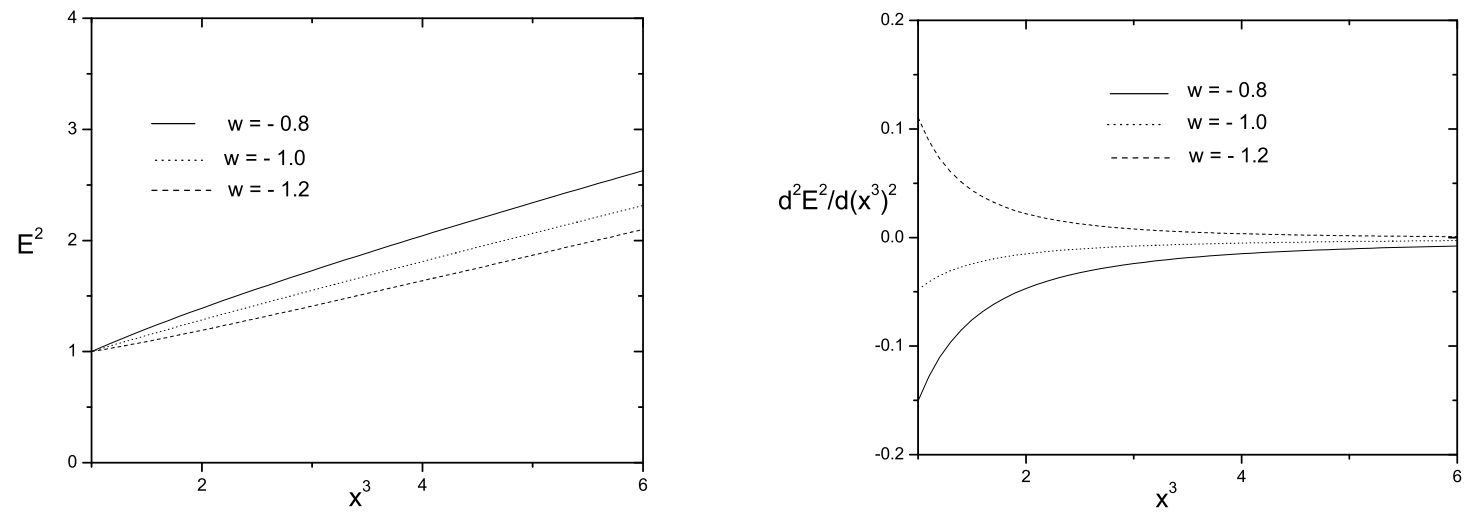

FIG. 3: Left panel: Graphs of $E^{2}$ vs. $x^{3}$ as given by Eq. (30) for three values of $w$. Right panel: Graphs of the second derivative of $E^{2}$ with respect to $x^{3}$ for the same three values of $w$. Notice that only phantom models present convex curves for reasonable values of the parameters. In drawing the graphs we took $\Omega_{b 0}=0.04, \Omega_{c 0}=0.26, \Omega_{d 0}=0.70$, and $\epsilon=0.05$.

Figure 4 shows the evolution of $E^{2}$ for the best fit value of model IV $(\epsilon=0.0006)$ of [34] (dotted line) and the values corresponding to the $1 \sigma$ error, $\epsilon=0.0011$ (dashed) and $\epsilon \simeq 0$ (solid); obviously the latter practically coincides with the noninteracting case. Likewise, Fig. 5 shows the evolution of $d^{2} E^{2}(x) / d\left(x^{3}\right)^{2}$, for the best fit values of the same model (dotted line) and for the noninteracting case (solid line). As is apparent the graphs practically overlap. 


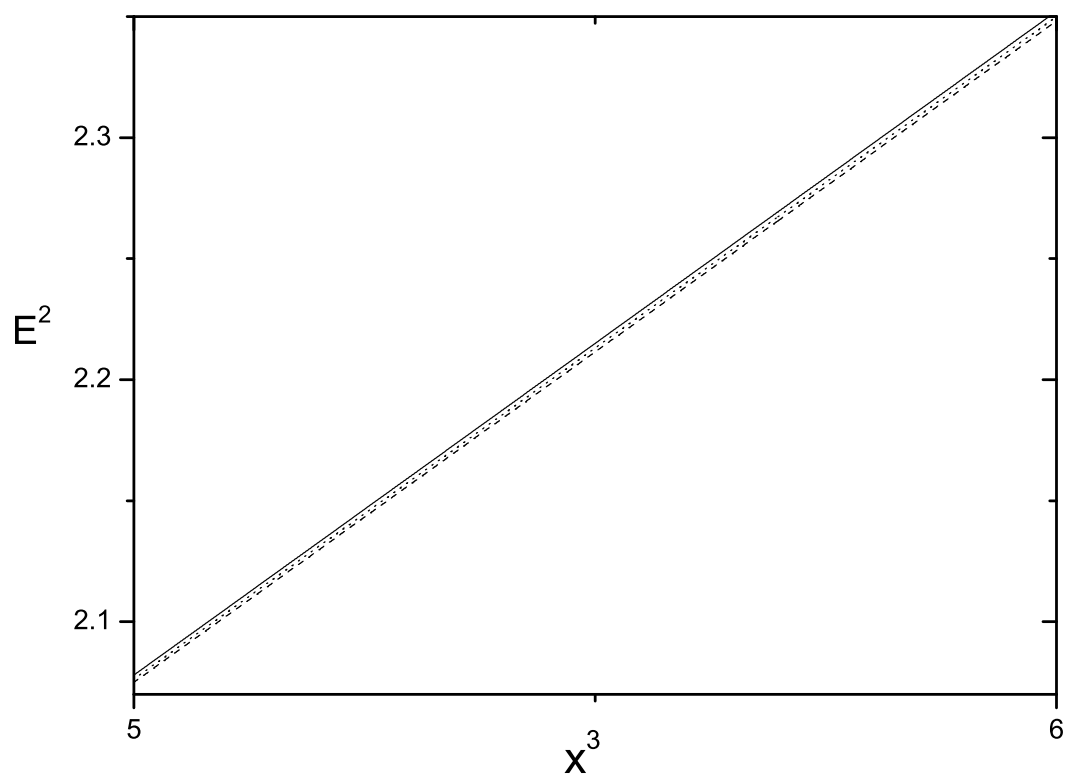

FIG. 4: Dotted line: plot of $E^{2}$ in terms of $x^{3}$ for the best fit values, $w=-1.03, \Omega_{c 0}=0.230$, and $\Omega_{d 0}=0.725, \epsilon=0.0006$, (dotted line) and the $\epsilon$ values corresponding to the $1 \sigma$ errors, 0.0011 (dashed) and $\simeq 0$ (solid) of model IV, $Q=3 H \epsilon\left(\rho_{c}+\rho_{d}\right)$, of Ref. 34]. We just depict the $5 \leq x^{3} \leq 6$ interval because the difference between the interacting and noninteracting case augments with redshift.

\section{INTERACTION TERMS OF THE TYPE $Q=Q\left(\rho_{c}, \rho_{d}\right)$}

In case $(i i)$-i.e., for $Q$ given by Eq. (6) - analytical solution can be found, at most, for one of the conservation equations, either (4) or (5), and this only if $\Gamma_{d}=0$, or $\Gamma_{c}=0$. Hence, at least one these equations must be solved numerically whereby no analytical expression exists for the Hubble factor. This limits the usefulness of $H(z)$ to set diagnostics on the nature of DE.

Let us consider first the case $\Gamma_{c}=0$ and $\Gamma_{d}>0$. In this instance, the currently phase of DE domination would be a transient one, the smaller $\Gamma_{x}$, the longer the DE domination.

Left and right panels of panel of Fig. [6] depict the evolution of $E^{2}$ and its second derivative in terms of $x^{3}$ for $\Gamma_{d}=0.1 H_{0}$.

As it can be seen, $d^{2} E^{2}(x) / d\left(x^{3}\right)^{2}$ presents a very fast increase (decrease) for phantom and 


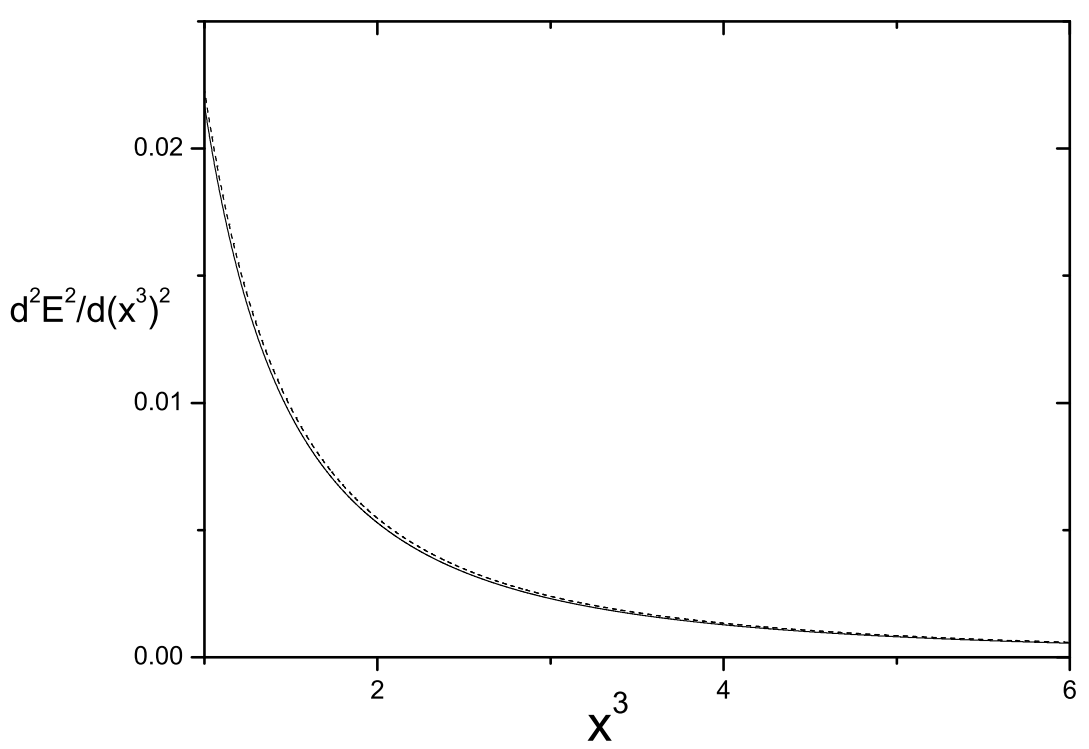

FIG. 5: Dotted line: plot of the second derivative of $E^{2}$ for the best fit values, $w=-1.03$, $\epsilon=0.0006$, with $\Omega_{c 0}=0.230$, and $\Omega_{d 0}=0.725$, of model IV, $Q=3 H \epsilon\left(\rho_{c}+\rho_{d}\right)$, of Ref. [34]. Solid line: same as before but with $\epsilon \simeq 0$ (no interaction).

DCC (for quintessence) at small redshifts $(z \ll 1)$. This feature is absent in noninteracting models as well as in models whose interaction term is of the general type considered in the previous section.

As a second example, let us consider the interaction term given by Eq. (6) with $\Gamma_{c}=\Gamma_{d}=$ $0.1 H_{0}$. As can be seen in Fig. 7, the behavior of the second derivative, $d^{2} E^{2}(x) / d\left(x^{3}\right)^{2}$, at small redshifts is very similar to the previous case whence we cannot tell one interaction from the other. Accordingly, an initial steep slope followed by an abrupt change to a much milder slope appears to be a general characteristic of interacting models in which $Q$ is proportional to the densities of DM or DE or to a combination of them. We hope this will serve to discriminate this set of interactions from the rest. 

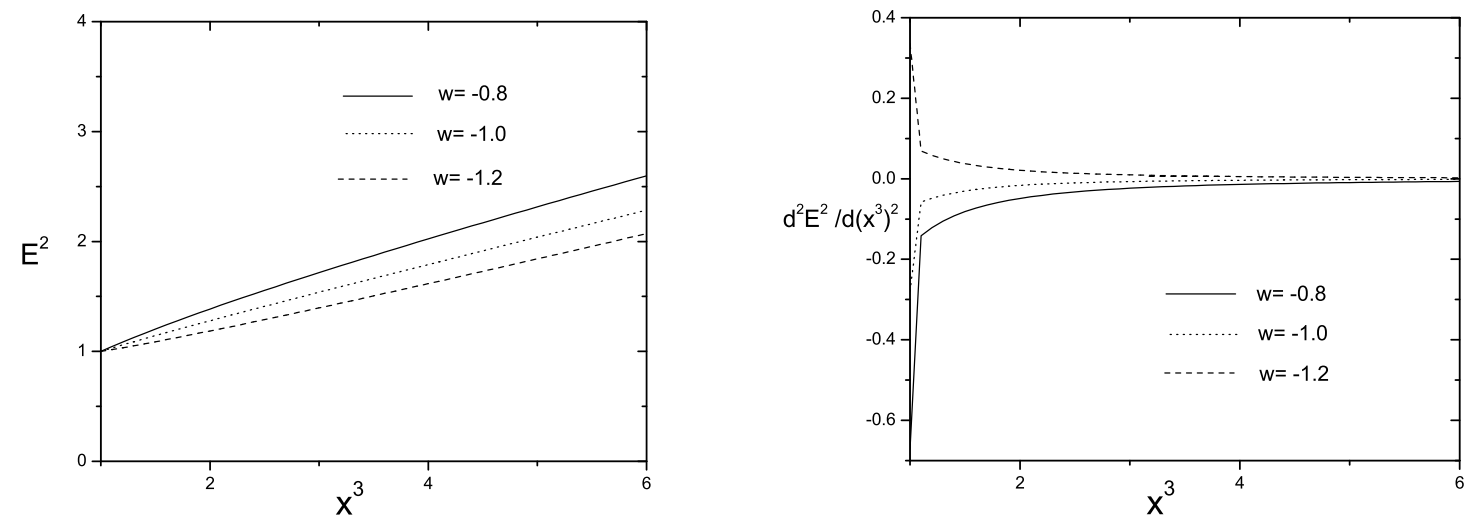

FIG. 6: Left panel: Graphs of $E^{2}$ vs. $x^{3}$ assuming $Q=3 \Gamma_{d} \rho_{d}$ with $\Gamma_{d}=0.1 H_{0}$ for three values of $w$. Right panel: Graphs of the second derivative of $E^{2}$ with respect to $x^{3}$ for the same three values of $w$. In drawing the graphs we took $\Omega_{b 0}=0.04, \Omega_{c 0}=0.26$, and $\Omega_{d 0}=0.70$.
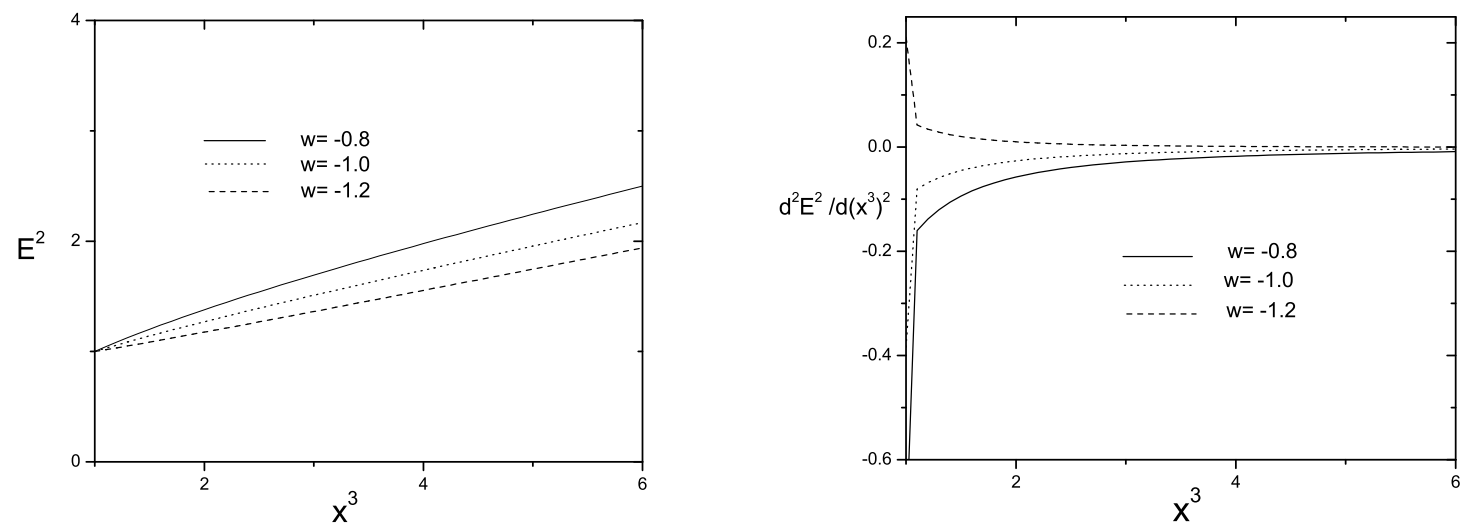

FIG. 7: Left panel: Graphs of $E^{2}$ vs. $x^{3}$ assuming $Q=3\left(\Gamma_{c} \rho_{c}+\Gamma_{d} \rho_{d}\right)$ with $\Gamma_{c}=\Gamma_{d}=0.1 H_{0}$ for three values of $w$. Right panel: Graphs of the second derivative of $E^{2}$ with respect to $x^{3}$ for the same three values of $w$. In drawing the graphs we took $\Omega_{b 0}=0.04, \Omega_{c 0}=0.26, \Omega_{d 0}=0.70$.

\section{THE STATEFINDER PARAMETERS}

Years ago Sahni et al. [38] and Alam et al. [39]-based on the dimensionless, geometrical pair of parameters $R$ and $S$ - gave a somewhat similar diagnostic of dark energy. These are defined in terms of the third temporal derivative of the scale factor, namely, $R=\dddot{a} /\left(a H^{3}\right)$ 
and $S=(R-1) /\left(3\left(q-\frac{1}{2}\right)\right)$, where $q$ stands for the deceleration parameter. The usefulness of the said pair stems from the fact that different models are characterized by different graphs in the $(S, R)$ plane; in particular, the $\Lambda$ CDM model is associated to the single point $(0,1)$. So, they may help elucidate the right DE candidate.

When these parameters are calculated for cosmological models in which DE and DM interact with each other it is seen that the interaction first appears in the third derivative of the scale factor [40]. In particular, for $Q=3 \epsilon H \rho_{c}$ one has

$$
R=1+\frac{9}{2} w\left(1+w \Omega_{d}\right) \frac{(1+w) \Omega_{d}+\epsilon \Omega_{c}}{\Omega_{b}+\Omega_{c}+(1+w) \Omega_{d}} .
$$

More specifically if one wished to resort to the present value of $R$ to discriminate the $\Lambda \mathrm{CDM}$ model from the DCC model $\left(w=-1, Q=3 \epsilon H \rho_{c}\right)$ of sub-section III.A, one would find $R_{0}=1-\frac{9}{2} \epsilon \Omega_{c 0}$.

If, instead, the interaction is governed by Eq. (6) one obtains

$$
R=1+\frac{9}{2} w\left(1+w \Omega_{d}\right) \frac{(1+w) \Omega_{d}+\frac{1}{H}\left(\Gamma_{c} \Omega_{c}+\Gamma_{d} \Omega_{d}\right)}{\Omega_{b}+\Omega_{c}+(1+w) \Omega_{d}} .
$$

This suggests that once the $\Omega$ parameters come to be accurately determined at different redshifts, the statefinder parameters will not only tell whether the interaction really exists but also will help discern the expression for $Q$. However, for the time being, the wide uncertainties about these seriously restrict the utility of the $R, S$ pair in this respect.

Altogether, the diagnostics founded on the $E^{2}$ and $d E^{2} / d x^{3}$ functions appear more advantageous since the interaction parameters (i.e., the $\epsilon$ and $\Gamma_{i}$ quantities), enter the Hubble factor upon integration (analytical or numerical) of the conservation equations (3) $-(5)$.

\section{DISCUSSION}

Interacting models of dark energy are well motivated, substantially alleviate the coincidence problem, and show compatibility with observation. Thus the question arises, "can they be discriminated from noninteracting models?" For the time being, the answer is not given our still imperfect knowledge of basic cosmological parameters, such as the Hubble factor. The latter is key in constraining models with observational data from baryon acoustic oscillations, the R-shift parameter of cosmic background radiation, the matter growth parameter, etc. Nevertheless, the situation is to improve greatly in the not far future thanks to a variety of upcoming and planed experiments. 
To extract the invaluable information about the nature of dark energy encrypted in the history of the Hubble factor is does not suffice to have abundant, accurate data of $H(z)$. One must first set up simple and practical criteria to be used once these data become available. We proposed several criteria, of notable mathematical simplicity, based on an accurate knowledge of the said history -something we reasonably hope to have at our disposal not very soon but, at least, in the foreseeable future.

Specifically if $d^{2} E^{2} / d\left(x^{3}\right)^{2}$ results positive (negative), then the dark energy is of phantom type (either quintessence or a decaying cosmological constant). Only if the DE is notdecaying cosmological constant, the graph of $E^{2}$ vs. $x^{3}$ will yield a straight line.

To discern whether DE is interacting (with $Q=Q\left(H \rho_{c}, H \rho_{d}\right)$ ) or not the behavior of $d E^{2}(x) / d x^{3}$ at high redshifts must be studied. If this derivative tends to the present value of the fractional baryon density, $\Omega_{b 0}$, then there will be grounds to believe that is it interacting with the interaction term $Q$ given either by Eq. (77) or (28). If it tends to $\Omega_{m 0}$, then we may strongly suspect that it is not interacting; and, in particular, if it coincides with $\Omega_{m 0}$ independently of redshift, then it will most likely be a conserved cosmological constant -see Eq. (11). Finally, if the redshift function $d E^{2}(x) / d x^{3}$ does not tend to any of these two quantities, we may conclude that either DE is interacting with $Q$ given by Eq. (21) or some other law not considered here, or that in reality $w$ is not a constant but a function of redshift. Obviously, this would complicate matters very much because it would call for the introduction of further parameters in the analysis -something that we defer to a future work.

If the plot of $d^{2} E^{2}(x) / d\left(x^{3}\right)^{2}$ vs. $x^{3}$ exhibits a very steep slope at redshifts much smaller than unity, we may conclude that the interaction obeys Eq. (6) (with $\Gamma_{c}$ and $\Gamma_{d}$ nonnegative). Its absence excludes such interaction, but it is very hard to specify whether the slope is steep enough. On the other hand, since this criterion deals with the slope of the second derivative extremely accurate data of $H(z)$ at these redshifts must be employed.

The statefinder parameters [38 40] will be very useful to rule out cosmological models, and tell apart different interacting models, but first the histories of $\Omega_{c}(z)$ and $\Omega_{d}(z)$ must be accurately established in a model-independent manner. This looks more challenging than obtaining an accurate history of the Hubble factor.

Since the interaction alters the abundance of dark matter in the past two additional ways to ascertain if the DE is interacting or not can be implemented, namely, to examine the 
behavior of the growth factor, and to study the weak lensing power spectrum. If -as we assumed throughout- the DE decays into dark matter, then it must have been less dark matter in the past than in the corresponding noninteracting model with the same $w$ and, consequently, the growth factor must be smaller. On the other hand, this would imply a decline of the lensing spectrum (the opposite would hold true if DM decayed into DE). Both

effects have been considered by the authors of Ref. [41]. However, they do not take $w$ constant but assume the Chevalier-Polarski-Linder ansatz [42] with very specific values for the parameters entering that expression. This is why comparison with our work does not seem straightforward. At any rate, both studies may be seen as complementary.

While the criteria introduced in this paper should be helpful in deciphering the nature of DE they should be used alongside the analysis and interpretation of data coming from a variety of sources: supernovae type Ia, cosmic microwave background radiation (CMBR), matter power spectrum, baryon acoustic oscillations, weak lensing, and evolution of galaxy clusters. The latter lies at the core of recent studies about the dynamical equilibrium of the clusters which seem to favor a decay of DE into DM over other possible scenarios [43].

In particular, a promising way to test the existence of the interaction is to consider its impact on the integrated Sachs-Wolfe effect. Since the former alters the evolution rate of gravitational potentials it must affect the frequency shift experienced by CMBR photons crossing collapsing structures. Again, studies of this kind hint at the existence of the interaction with DE decaying into DM [44, 45].

We restricted ourselves to constant $w$ and vanishing spatial curvature. While this automatically limits the scope of our work we feel it does not do it seriously, at least no more than otherwise since it would introduce additional unknown parameters that would compromise the said scope in other ways.

\section{Acknowledgments}

Thanks are due to the anonymous refreee and Fernando Atrio-Barandela for constructive comments and criticisms on a earlier draft of this paper, and Eric Linder and Craig Hogan for drawing our attention to Refs. [10] and [11], respectively. This work was supported from "Comisión Nacional de Ciencias y Tecnología" (Chile) through the FONDECYT Grant No. 1070306 (SdC) and No. 1090613 (RH). D.P. acknowledges "FONDECYT-Concurso incentivo 
a la cooperión internacional" No. 7100025, and is grateful to the "Instituto de Física" for warm hospitality; also D.P. research was partially supported by the "Ministerio Español de Educación y Ciencia” under Grant No. FIS2006-12296-C02-01.

[1] V. Sahni, A. Shafieloo, and A.A. Starobinsky, Phys. Rev. D 78, 103502 (2008).

[2] P. Serra, A. Cooray, D.E. Holz, A. Melchiorri, S. Pandolfi, and D. Sarkar, Phys. Rev. D 80, $121302(\mathrm{R})(2009)$.

[3] P.J.E. Peebles and B. Ratra, Rev. Mod. Phys. 75, 559 (2003); K. Hagiwara et al., Phys. Rev. D 66, 010001(2002).

[4] C. Wetterich, Nucl.Phys. B 302, 668 (1988); W. Zimdahl, D. Pavón, and L.P. Chimento, Phys. Lett. B 521, 133 (2001); L. Amendola and D. Tocchini-Valentini, Phys. Rev. D 66, 043528 (2002); G. Farrar and P.J.E. Peebles, Astrophys. J 604, 1 (2004); S. Das, P.S. Corasaniti, and J. Khoury, Phys. Rev. D 73, 083509 (2006); S. del Campo, R. Herrera, and D. Pavón, Phys. Rev. D 74, 023501 (2006); R. Horvat, Phys. Lett. B 648, 347 (2007); R. Manini and S. Bonometto, JCAP 06(2007)020; Z.-K. Guo, N. Ohta, and S. Tsujikawa, Phys. Rev. D. 76, 023508 (2007); F.E.M. Costa, J.S. Alcaniz, and J.M.F. Maia, Phys. Rev. D 77, 083516 (2008); G. Izquierdo and D. Pavón, Phys. Lett. B 688, 115 (2010); F. Atrio-Barandela and D. Pavón, "Interacting dark energy" in Dark Energy-Current Advances and Ideas, edited by J.R. Choi (Research Signpost, Trivandrum, Kerala, India, 2010); E. Abdalla, R. Abramo, and J.C.C. de Souza, Phys. Rev. D 28, 023508 (2010).

[5] A.M. Polyakov, Nucl.Phys. B 797, 199 (2008).

[6] A.M. Polyakov, Nucl.Phys. B 834, 316 (2010).

[7] J. Simon, L. Verde and R. Jiménez, Phys. Rev. D 71, 123001 (2005).

[8] R.A. Daly, S.G. Djorgovski, K.A. Freeman, M. Mory, C.P. O’Dea, P. Kharb, and S. Baum, Astrophys. J. 677, 1 (2008).

[9] E. Gaztañaga, A. Cabré, and L. Hui, Mon. Not. R. Astron. Soc. 399, 1663 (2009).

[10] D. Schlegel, M. White, and D. Eisenstein, arXiv:0902.4680 [astro-ph.CO].

[11] C. Hogan, Phys. Rev. D 77, 043512 (2008).

[12] C. Hogan, personal communication.

[13] P.-S. Corasaniti, D. Huterer, and A. Melchiorri, Phys. Rev. D 75, 062001 (2007). 
[14] A. Loeb, Astrophys. J. 499, L111 (1998).

[15] S. del Campo, R. Herrera, and D. Pavón, Phys. Rev. D 78, 021302(R) (2008); ibid JCAP 01(2009)020.

[16] D. Pavón and B. Wang, Gen. Relativ. Grav. 41, 1 (2009).

[17] If it were large and positive, DM would dominate the expansion today. On the other hand, if $Q$ were large and negative, the Universe would have been dominated by DE practically from the outset and galaxies would not have come into existence.

[18] G. Caldera-Cabral, R. Maartens, and L.A. Ureña-López, Phys. Rev. D 79, 063518 (2009).

[19] N. Kaloper and K.A. Olive, Phys. Rev. D 57, 811 (1988).

[20] R. Curbelo, T. González, and I. Quirós, Class. Quantum Grav. 23, 1585 (2006).

[21] H. Zhang and Z.-H. Zhu, Phys. Rev. D 73, 043518 (2006).

[22] M.S. Turner, Phys. Rev. D 28, 1243 (1983).

[23] K.A. Malik, D. Wands, and C. Ungarelli, Phys. Rev. D 67, 063516 (2003).

[24] R. Cen, Astrophys. J. 546, L77 (2001).

[25] J.M. Overduin and F.I. Cooperstock, Phys. Rev. D 58, 043506 (1998).

[26] I. L. Shapiro, J. Solá, C. España- Bonet, and Ruiz-Lapuente, Phys. Lett. B 574, 149 (2003).

[27] C. España-Bonet, P. Ruiz-Lapuente, I.L. Shapiro, andJ. Solá, JCAP 02(2004)006.

[28] P. Wang and X.H. Meng, Class. Quantum Grav. 22, 283 (2005).

[29] M. Maggiore, arXiv:1004.1782v2 [astro-ph.CO].

[30] N. Bilic, arXiv:1004.4984v2 [hep-th].

[31] J.C. Fabris, I.L. Shapiro, and J. Solá, JCAP 02(2007)016.

[32] S. Basilakos, M. Plionis, and J. Solá, Phys. Rev. D 80, 083511 (2009).

[33] K.A. Olive, "TASI lectures on dark matter", arXiv:astro-ph/0301505.

[34] J.-H. He, B. Wang, and E. Abdalla, "Testing the interaction between dark energy and dark matter via latest observations", arXiv:1012.3904.

[35] S. Hansen, J. Lesgourgues, S. Pastor, and J. Silk, Mon. Not. R. Astr. Soc. 333, 544 (2002).

[36] J. Zhou, Bin Wang, Pavón, and E. Abdalla, Mod. Phys. Lett. A 24, 1689 (2009).

[37] L.P. Chimento, A.S. Jakubi, D. Pavón, and W. Zimdahl, Phys. Rev. D 67, 083513 (2003); B. Wang et al. Phys. Lett. B 637, 357 (2006); G. Olivares, F. Atrio-Barandela, and D. Pavón, Phys. Rev. D 77, 063513 (2008).

[38] V. Sahni, T.D. Saini, A.A. Starobinsky, and U. Alam, JETP Lett. 77, 201 (2003). 
[39] U. Alam, V. Sahni, T.D. Saini, and A.A. Starobinsky, Mon. Not. R. Astron. Soc. 344, 1057 (2003).

[40] W. Zimdahl and D. Pavón, Gen. Relativ. Grav. 36, 1483 (2004).

[41] G. Caldera-Cabral, R. Maartens, and B.M. Schaefer, JCAP 07(2009)027.

[42] M. Chevallier and D. Polarski, Int. J. Mod. Phys. D 10, 213 (2001); E.V. Linder, Phys. Rev. Lett. 90, 091301 (2003).

[43] E. Abdalla, L.R. Abramo, L. Sodré Jr., and B. Wang, Phys. Lett. B 673, 107 (2009).

[44] G. Olivares, F. Atrio-Barandela and D. Pavón, Phys. Rev. D 77, 103520 (2008).

[45] J.-H. He, B. Wang, and P. Zhang, Phys. Rev. D 80, 063530 (2009). 